\section{\$38. Molecular Dynamics Simulation for Structure Formation of a Single Polymer Chain in Solution}

Fujiwara, S., Hashimoto, M., Itoh, T. (Kyoto Inst. Tech.)

Nakamura, $\mathrm{H}$.

Structure formation of polymer chains such as polymer crystallization has so far been extensively studied. Since polymer chains have many internal degrees of freedom, they show diverse static and dynamical structures. Because of their structural diversity, polymeric materials have various physical properties. Over the last decade, many studies have been done on the polymer crystallization by molecular dynamics (MD) simulation. However, owing to the large computational effort necessary, few MD works have been made on the structure formation of a single polymer chain immersed in a bath of solvent molecules. The purpose of this work is to clarify, at the molecular level, the mechanisms of the structure formation of a single polymer chain in solution. To this end, we carry out the MD simulations of a single polymer chain immersed in a bath of solvent molecules and analyze the formation process of the orientationally ordered structure during quenching.

The computational model is the same as that used in our previous works ${ }^{1), 2)}$. We consider a linear polymer chain with 500 methylene groups immersed in 3747 n-alkanes with six methylene groups. The methylene group is treated as a united atom. The united atoms interact via bonded potentials (bond-stretching, bondbending and torsional potentials) and a non-bonded potential (12-6 Lennard-Jones (LJ) potential). The atomic force field used here is the DREIDING potential ${ }^{3}$. The equations of motion for all atoms are solved numerically using the velocity Verlet algorithm. We use the combination of a constant-temperature method ('ad hoc' velocity scaling method) and a constant-pressure method (Andersen's method). We apply the periodic boundary conditions. The MD simulations are performed as follows. At first, we provide a randomly distributed conformation of polymer solution in thermal equilibrium at high temperature $(T=550 \mathrm{~K})$. The system is then quenched to several lower temperatures $(T=300,350,400$ and $450 \mathrm{~K}$ ). A simulation of $10 \mathrm{~ns}$ is carried out at each temperature.

In comparison with the simulation results of an isolated polymer chain in vacuum, it is ascertained that the stem length of the orientationally ordered structure formed in solution becomes longer than that formed in vacuum ${ }^{1)}$. In order to elucidate the reason why the stem length in solution is longer than that in vacuum, we make an energy analysis of a polymer chain in solution. The bonded potential energy, $E_{\mathrm{b}}$, in the case of longer stems in solution is smaller than that in the case of shorter stems in vacuum (Fig.1(a)). The LJ potential energy associated with a polymer chain is divided into two parts: one is the LJ potential energy between the constituent atoms of a polymer chain, $E_{\mathrm{pp}}$, and the other is that between the constituent atoms of a polymer chain and solvent molecules, $E_{\mathrm{ps}}$. We show, in Figs.1(b) and $1(\mathrm{c}), E_{\mathrm{b}}+E_{\mathrm{pp}}$ and $E_{\mathrm{b}}+E_{\mathrm{pp}}+E_{\mathrm{ps}}$ in the case both of longer stems and of shorter stems. Figure 1(b) tells us that $E_{\mathrm{b}}+E_{\mathrm{pp}}$ in the case of longer stems in solution is larger than that in the case of shorter stems in vacuum. This fact indicates that the orientationally ordered structure with longer stems is energetically more unstable than that with shorter stems if the van der Waals interaction between a polymer chain and solvent molecules is not taken into account. We find from Fig.1(c) that $E_{\mathrm{b}}+E_{\mathrm{pp}}+E_{\mathrm{ps}}$ in the case of longer stems is smaller than that in the case of shorter sterns, that is, in solution the orientationally ordered structure with longer stems is energetically more stable than that with shorter stems because of the van der Waals interaction of a polymer chain with the surrounding solvent molecules.
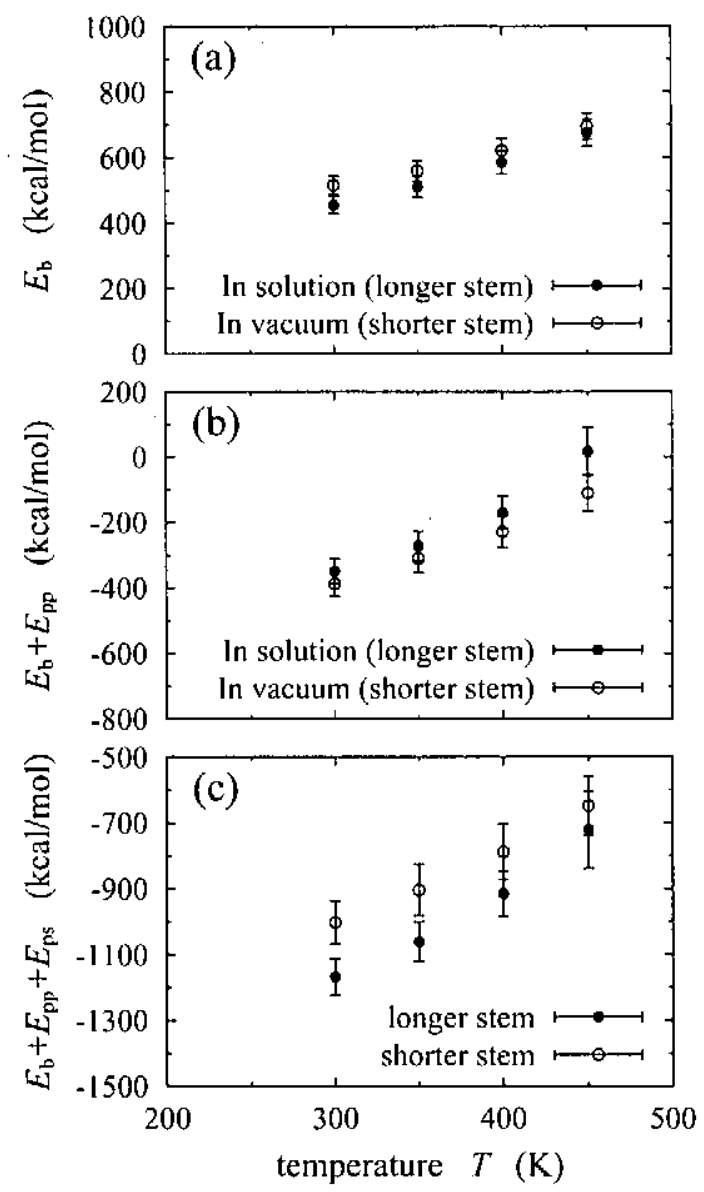

Fig. 1. The temperature dependence of the potential energies in the cases of longer stems and shorter stems: (a) the bonded potential energy $E_{\mathrm{b}}$, (b) the bonded and LJ potential energy $E_{\mathrm{b}}+E_{\mathrm{pp}}$, and (c) the bonded and LJ potential energy $E_{\mathrm{b}}+E_{\mathrm{pp}}+E_{\mathrm{ps}}$.

\section{References}

1) Fujiwara, S. and Sato, T., Comput. Phys. Commun. 147, (2002) 342.

2) Fujiwara, S. and Sato, T., J. Macromol. Sci. - Phys. B42, (2003) 455.

3) Mayo, S.L. et al., J. Phys. Chem. 94, (1990) 8897. 